\title{
Angiosarcoma cardíaco metastásico, presentación inusual en paciente femenina de 35 años de edad
}

- Carolina López Ordóñez, Esteban Castro Paz, Eider Felipe Moreno Cortés, Yorlany Rodas Cortés, Juan Paulo Martínez

Hemato Oncólogos S.A., Cali (Valle del Cauca).

Contacto: tekztro@gmail.com

Introducción y objetivos. El angiosarcoma cardíaco es un tumor primario, maligno e infrecuente, extensamente infiltrativo en estructuras cardíacas. Se origina generalmente en aurícula derecha y pericardio, común en varones. Los tumores cardíacos primarios son raros y su incidencia oscila entre el 0,0017-0,003\%. El angiosarcoma representa un $31 \%$ de los tumores malignos primarios cardíacos.

Materiales y métodos. Paciente femenina de 35 años, consulta por dolor epigástrico y palpitaciones. El examen físico reveló desdoblamiento del S1 y hepatomegalia. Se inician estudios documentando lesiones hepáticas y esplénicas metastásicas asociadas a masa auricular derecha acompañada de nodulaciones intracardíacas con probable compromiso pericárdico.

Resultados. La paciente es intervenida quirúrgicamente con escisión de tumor cardíaco. Se realizaron estudios histológicos e inmunohistoquímicos con diagnóstico compatible con angiosarcoma cardíaco. La paciente se encuentra en tratamiento de quimioterapia con paclitaxel semanal, lleva cuatro ciclos con aceptable tolerancia.

Conclusiones. La importancia radica en la baja incidencia reportada en la literatura. La inmunohistoquímica es determinante para el diagnóstico.

\section{Paraganglioma extraadrenal en mujer de 22 semanas de gestación}

- Carolina López Ordóñez, Eider Felipe Moreno Cortés, Esteban Castro Paz, Yorlany Rodas Cortés, Iván Bravo

Hemato Oncólogos S.A., Cali (Valle del Cauca).

Contacto: eidermc94@gmail.com

Introducción y objetivos. Los paragangliomas son neoplasias poco frecuentes, malignidad rara definida por existencia de metástasis, incidencia 0,2-0,5:100.000 hab./año. El tratamiento esencial en cualquier localización es cirugía.

Materiales y métodos. Paciente femenina de 26 años, sin antecedentes relevantes. Refirió dolor lumbar severo con alteraciones para la locomoción, embarazo de 22 semanas; por severidad de los síntomas, requirió RMN abdominal/lumbosacra (sin contraste), evidenció lesión lítica en L1 produciendo estrechez del cordón y masa retroperitoneal comprimiendo vena cava inferior. Requirió interrupción del embarazo.

Resultados. Le realizan resección de lesión a nivel lumbar/estabilización de columna. Patología e inmunohistoquímica corroboran diagnóstico de paraganglioma. Cromogranina y OctreoScan positivos. Actualmente en manejo con análogos de somatostatina.

Conclusiones. El paraganglioma es una enfermedad rara cuyo diagnóstico es difícil por su localización y por la ausencia de síntomas específicos y de marcadores bioquímicos. Este caso se presentó como dolor lumbar severo en mujer gestante. La inmunohistoquímica es fundamental para corroborar el diagnóstico. 